\title{
The Monoclonal, Massive Globulin- Waldenstrom Macroglobulinaemia
}

\author{
Anubha Bajaj* \\ Department of Diagnostics, Punjab University, India \\ Submission: January 24, 2019; Published: February 27, 2019 \\ *Corresponding author: Anubha Bajaj, Department of Pathology, Punjab University, Chandigarh, India
}

\section{Preface}

Wald Enstrom macrogobulinaemia is a disorder designated withanomenclature ofa Swedish physicianJan GostaWaldenstrom (1906-1996). The exceptional disease was initially scripted in 1944 [1,2]. Waldenstrom macroglobulinaemia may be defined as the appearance of a serum para-protein such as immunoglobulin M (Ig M) in addition to a malignant lymphoplasmacytic infiltrate confined to the bone marrow. Lymphoplasmacytic Lymphoma (LPL) may be cogitated as a neoplasm comprising of miniature B lymphocytes, plasmacytoid lymphocytes and mature plasma cells. The tumefaction generally implicates the bone marrow with an occasional presence in the lymph node and spleen. Lymphoplasmacytic lymphoma is accompanied by Waldenstrom macroglobulinaemia in a majority $(95 \%)$ of instances $[1,2]$. The dual conditions may be denominated by an immunoglobulin M (Ig M) monoclonal gammopathy accompanied by an emergence of a lymphoplasmacytoid lymphoma restricted to the bone marrow. Lymphoplasmacytoid lymphoma may concur with an infection of hepatitis C virus (HCV). A familial prevalence may be delineated. An estimated $1.4 \%$ of neoplasm of miniature B lymphocytes may be cogitated by lymphoplasmacytoid lymphoma [1,2].

\section{Disease Characteristics}

Lymphoplasmacytic lymphoma may be categorized as a post germinal centre B cell (CD10-, MUM 1+ and BCL6+/-) lymphoma commingled with divergent plasmacytic cellular differentiation. The lymphoma may depict concomitant infection with Hepatitis $\mathrm{C}$ Virus (HCV). The bone marrow infiltrate may predominantly be interstitial, nodular or of a diffuse configuration. Bone marrow trephine biopsy and bone marrow aspirate may demonstrate an admixture of miniature lymphocytes, plasmacytoid lymphocytes and mature plasma cells $[2,3]$. The malignant cellular egress may enunciate a monotypic secretion of serum immunoglobulin $\mathrm{M}$ (Ig M) protein which may be elucidated in a majority (> 90\%) of instances. Subjects with Waldenstrom macroglobulinaemia frequently depict vascular hyper-viscosity. Thus, Waldenstrom macroglobulinaemia may be cogitated as a non-Hodgkin's lymphoma concomitant with lymphoplasmacytic lymphoma as majority (95\%) of subjects of lymphoplasmacytic lymphoma elucidate features of Waldenstrom macroglobulinaemia. The indolent lymphoplasmacytic lymphoma and concomitant Waldenstrom macroglobulinaemia may exemplify a disorder of obscure origin [2,3]. Associated aspects of probable disease insurgence may be

\section{Male sex}

2. Enhancing age of disease emergence with a median age of diagnosis at 65 years,

3. A racial predisposition in Caucasians

4. The concurrence of immunoglobulin M monoclonal gammopathy of undetermined significance (Ig M MGUS).

Waldenstrom macroglobulinaemia may progress to adjunctive B lymphocyte malignancies with an estimated proportion of $10 \%$ at 5 years, $18 \%$ at 10 years and $24 \%$ at 15 years of disease incurrence with an overall ratio of malignant conversion at $1.5 \%$ per year. The neoplasm also displays a familial preponderance and nearly $20 \%$ individual's manifest family members suffering from Waldenstrom macroglobulinaemia and associated B lymphocyte malignancies. Environmental factors such as exposure to radiation or agent orange, hazardous occupation with handling leather, rubber, paints, dyes and solvents, coexistent autoimmune disease and infection with hepatitis $\mathrm{C}$ virus (HCV) may be incriminated in the evolution of the malignancy $[3,4]$.

\section{Clinical Elucidation}

The circulation of serum monoclonal immunoglobulin M (Ig M) in Waldenstrom macroglobulinaemia may display characteristic constitutional symptoms with concurrent deposition of monoclonal immunoglobulin M (Ig M) protein in several body tissues with a consequent emergence of autoantibodies. Waldenstrom macroglobulinaemia may manifest systemic symptoms with an estimated serum monoclonal immunoglobulin M (Ig M) protein greater than 3 grams/ 
decilitre and a bone marrow ingress of malignant lymphoplasmacytoid cells greater than $20 \%$. Approximately one fourth (27\%) instances of Waldenstrom macroglobulinaemia may be asymptomatic, with anaemia in roughly $38 \%$ subjects, the emergence of hyper-viscosity in around 31\% individuals, the appearance of B symptoms (fever, weight loss, night sweats) in nearly $23 \%$ and neurological symptoms in about $22 \%$ of patients $[1,2]$. Waldenstrom macroglobulinaemia may depict specific complications such as hyper-viscosity, tissue aggregation of immunoglobulin M (Ig M) or autoimmune haemolysis secondary to circulating macro-globulins. Subjects may present with haematemesis, haemorrhage from the nasal cavity and retinal vasculature [5,6]. Anaemia, thrombocytopenia, elevated Erythrocyte Sedimentation Rate (ESR), lymph node enlargement and hepato-splenomegaly may ensue. A bone marrow trephine biopsy may exemplify an abundance of malignant lymphoid cells. Radiographic analysis of the implicated bones may be unremarkable, thereby excluding a multiple myeloma. Serum protein examination may detect the presence of an extremely high molecular weight protein," a macroglobulin", cogitated as an excess of immunoglobulin M [5,6]. The quantification of monoclonal immunoglobulin M (Ig M) may be concordant with the magnitude of bone marrow infiltration and severity of systemic symptoms. Hyper- viscosity may appear as chronic haemorrhage from the nasal cavity, gingiva or gastrointestinal tract accompanied by headache, dizziness, loss of coordination or balance, impaired hearing with tinnitus with blurring or loss of vision. Retinopathy may ensue on account of distended retinal veins and swelling of the optic disc. Severely affected subjects may display manifestations of heart failure, drowsiness, stupor and coma. Systemic symptoms may be discerned at a quantifiable serum Ig M value greater than 4000 milligrams/decilitre, though immunoglobulin levels may vary. Constitutional or B symptoms such as fever, weight loss greater than $10 \%$ of the body weight in preceding six months, drenching night sweats and fatigue may appear [6,7]. Peripheral neuropathy may be a manifestation of the disorder. Cold agglutinin disease may occur on account of elevated circulating antibodies to red blood cells which may aggregate at minimal body temperatures and induce a haemolytic anaemia along with Raynaud's phenomenon, jaundice and haemoglobinuria. Cryoglobulinemia may be encountered with the precipitation of immunoglobulin M (Ig M) at reduced body temperatures in order to obstruct the miniature blood vessels with emerging consequences such as Raynaud's phenomenon, thrombocytopenic purpura, haemorrhaging ulcers and gangrene of the fingers, toes, nose and ears [1,2].

Amyloidosis may occur with the configuration of an anomalous "amyloid" protein" which may accumulate in tissues and organs of the body such as gastro-intestinal tract, renal and hepatic tissue or heart and peripheral nerves. Malfunctions such as carpal tunnel syndrome, malabsorption, macroglossia, dermal thickening, swelling of the extremities, congestive heart failure and renal failure may emerge. "BING NEEL" syndrome may be cogitated with a lymphoplasmacytic infiltrate or deposition of immunoglobulin M (Ig M) within the central nervous system (brain or spinal cord). Systemic symptoms such as mental deterioration, confusion, visual disturbance, irritability, altered personality, convulsions and coma may concur. Recurrent sinus and upper respiratory tract infection, pleural effusion, pulmonary infiltrates and occasional rash may be delineated. Tumour cells of lymphoplasmacytic lymphoma may configure nodular aggregates in the skin, extremities, spine, breast and orbital socket $[6,7]$.

\section{Morphological Elucidation}

Waldenstrom macroglobulinaemia with coexistent lymphoplasmacytic lymphoma enunciates malignant cells with characteristics of B lymphocytes and plasma cells, denominated as lymphoplasmacytic cells. A diffuse or interfollicular proliferation of malignant lymphoid cells may be cogitated. Cellular aggregates devoid of proliferation centres may be proportionately constituted by miniature $B$ lymphocytes, plasmacytoid lymphocytes and mature plasma cells. A predominant lympho-plasmacytic infiltrate may be situated in the inter-trabecular region of the bone marrow [3]. Peripheral blood picture concordant with acute leukaemia may be demonstrated in an estimated one third (30\%) instances. Tumour cells may predominantly omprise solely of miniature lymphocytes or small, mature lymphocytes commingled with plasmacytoid lymphocytes. The bone marrow may be infiltrated by an identical malignant infiltrate. Mature plasma cells, tissue mast cells and histiocytes may be quantifiably augmented. Plasma cells may infrequently be the preponderant cellular component. Serial sections from bone marrow trephine biopsy may delineate a diffuse or a focal lesion. The focal lesions may configure a para trabecular, interstitial or non paratrabecular pattern of tumour incrimination [3]. Expansive marrow replacement by the tumefaction may induce a significant reduction of normal haematopoiesis. Intra-nuclear inclusions termed as "Dutcher' s bodies" may be articulated within lymphocytes and plasma cells and may be considered diagnostic of lymphoplasmacytic lymphoma. Intra-nuclear inclusions reactive to Periodic Acid Schiff's (PAS) stain may similarly be configured in plasma cells constituting a multiple myeloma or reactive lymphoid proliferations. Lymphoplasmacytic lymphoma with Waldenstrom macroglobulinaemia may terminate with the evolution of a Richter's syndrome, thereby recapitulating a Small Lymphocytic Lymphoma (SLL). The malignant egress may lack the presence of monocytoid cells, in contrast to a marginal zone lymphoma. The occurrence of Dutcher 's bodies with admixed enlarged, transformed lymphocytes may be characteristic of lymphoplasmacytic lymphoma. Mast cells may be intermingled with epitheloid histiocytes. Intercellular material stained with periodic acid Schiff (PAS+) stain may be exemplified along with scattered amyloid and crystal engulfing histiocytes $[7,8]$. 


\section{Immune phenotype}

Lymphoplasmacytic lymphoma cells may exemplify a surface immunoglobulin M (Ig M+). Immunoglobulin molecules confined to the cytoplasm may primarily be immunoglobulin $\mathrm{M}$ (Ig M) although Ig G or infrequently Ig A may be elucidated. The lymphoma may be immune reactive to CD20+ and associated pan B lymphocyte antigens such as CD19+, CD79a+ and PAX5+. However, a percentage may be non eactive for the aforementioned immune markers. Nonreactive immune molecules may be CD5-, CD10-, CD23- and BCL6- although CD5 may be debatable (-/+) $(1,3)$. Plasmacytic immune markers CD38 and CD138 may be equivocal (+/-) in specific instances.

\section{Molecular Characterization}

A frequent genomic abnormality the MYD88L265P mutation may be discerned in a majority (95\%) along with chromosomal deletion of del [6] (q21). Chromosomal translocation t (9:14) may be exceptional. The MYD88L265P chromosomal mutation may be universal in Waldenstrom macroglobulinaemia. A whole genome sequencing may depict the mutation in $90 \%$ instances. MYD88L265P chromosomal mutation may be infrequent in multiple myeloma, marginal zone lymphoma or immunoglobulin M -monoclonal gammopathy of undetermined significance (Ig M MGUS). Chromosomal mutation CXCR4 may be enunciated which may be identical to the WHIM syndrome (warts, hypogammaglobulinaemia, infections and myelokathexis). Individuals with Waldenstrom macroglobulinaemia devoid of MYD88 or a CXCR4 chromosomal mutation may depict an inferior survival, in contrast to instances delineating the mutations $[1,2]$.

\section{Differential Diagnosis}

Lymphoplasmacytic lymphoma may necessitate a distinction from Chronic Lymphocytic Leukaemia (CLL), mantle cell lymphoma and plasmacytoid variants of extra nodal or nodal marginal zone lymphoma. Chronic Lymphocytic Leukaemia (CLL) may exhibit a focal plasmacytic differentiation. The tumour cells may be immune reactive to CD5+, CD23+ and a CD20 dim, in contrast to a lymphoplasmacytic lymphoma. Immunoglobulin $\mathrm{M}$ (Ig M) para-protein may be absent or minimal. Splenic marginal zone lymphoma may demonstrate an intra-sinusoidal pattern of marrow incrimination. Plasmacytic differentiation may be reduced or minimal. Immunoglobulin M (Ig M) para-protein may be lacking or be of miniscule quantities [1,2]. Distinction from plasmacytoid lymphoma may be particularly cogitated with demonstration of plasma cells and plasmacytoid lymphocytes, manifesting numerous inclusions confined to the cytoplasm which may react to the Periodic Acid Schiff 'S (PAS+) stain. The tumour cells may thus recapitulate the appearance of histiocytes. Chromosomal point mutation MYD88 may be elucidated in a majority (90\%) of instances of lymphoplasmacytic lymphoma, contrary to an exceptional delineation in multiple myeloma and marginal zone lymphoma [8,9] (Figures 1-14).
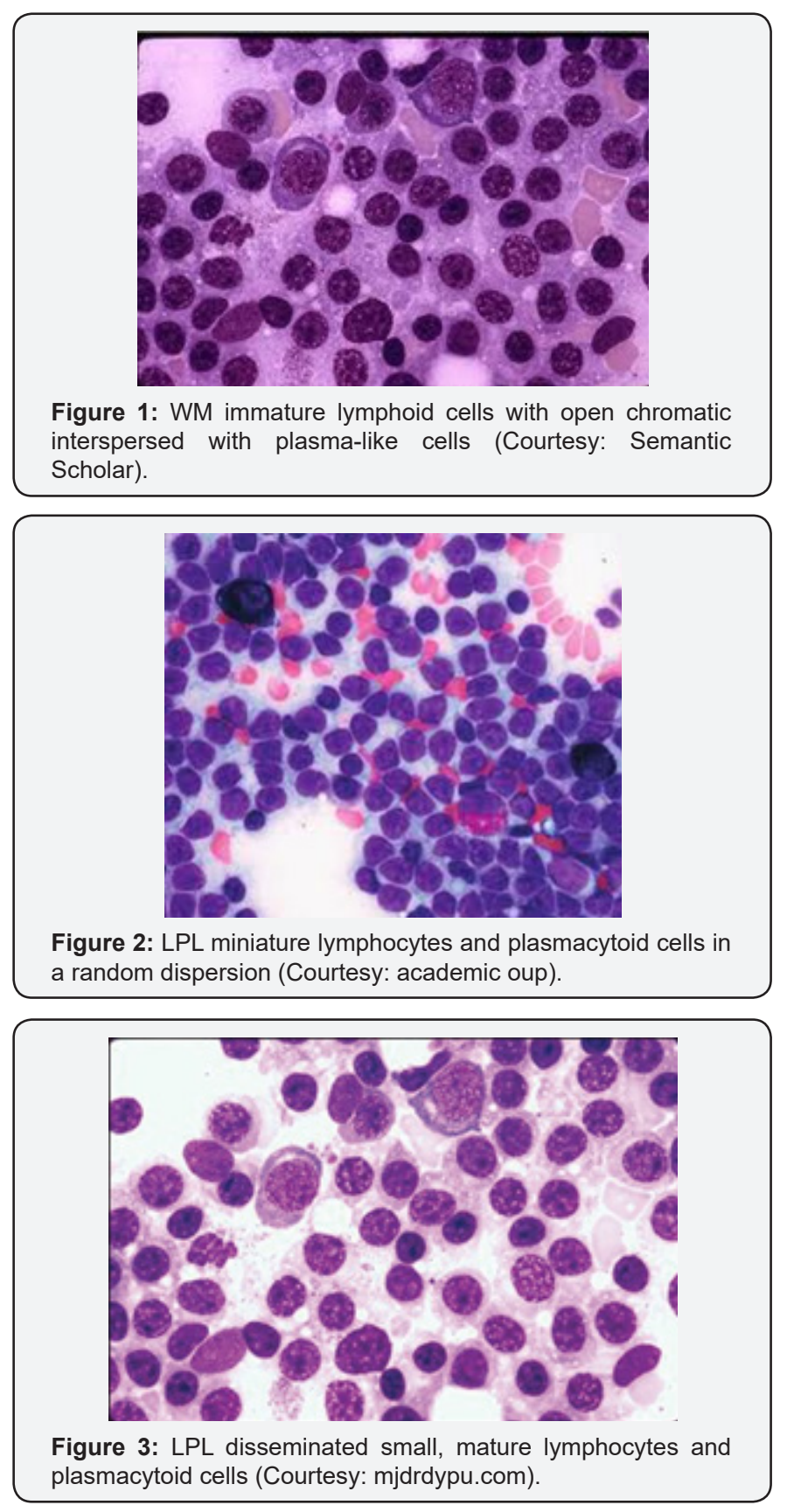

plasmacytoid cells (Courtesy: mjdrdypu.com).

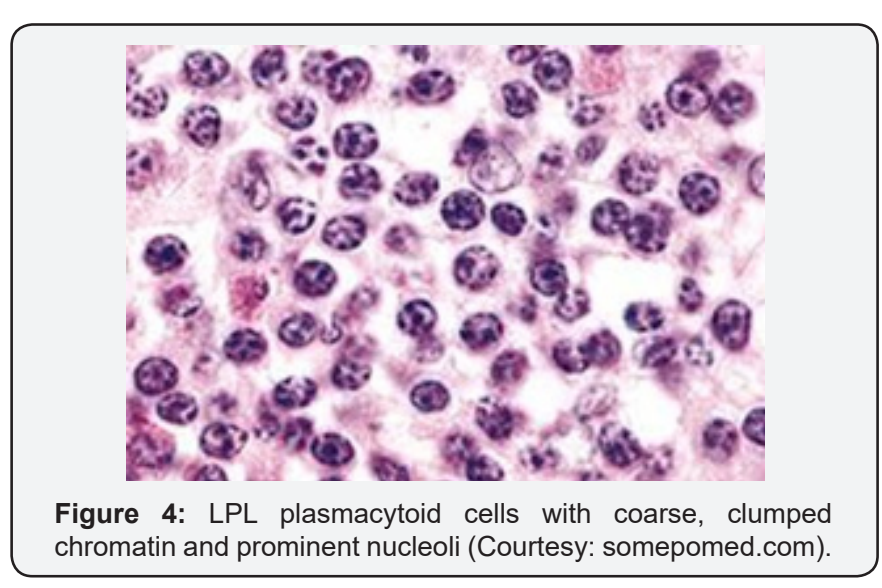




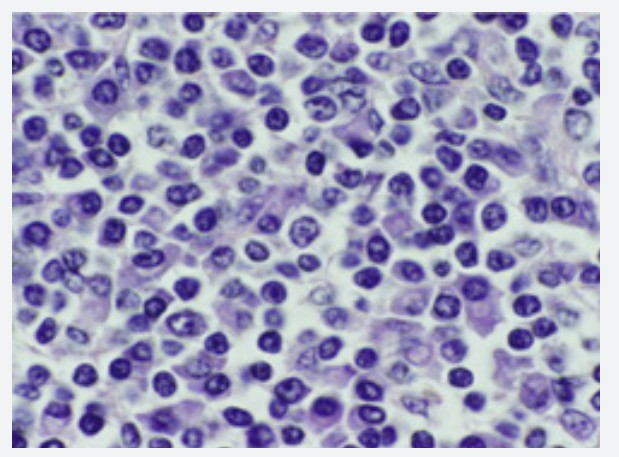

Figure 5: WM intense infiltrate of plasma cells and plasmacytoid cells (Courtesy: Science direct).

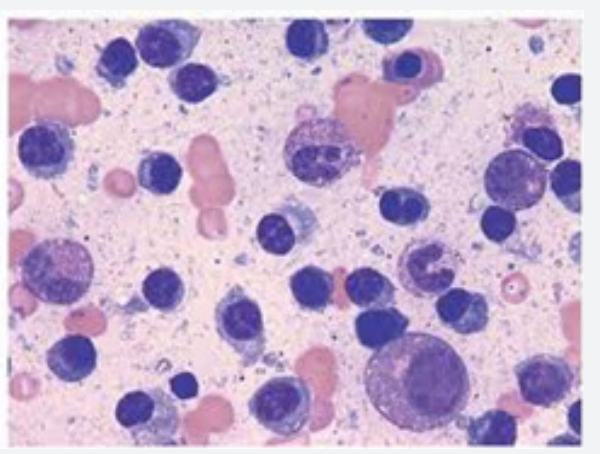

Figure 6: LPL vascular presence of plasma cells and plasmacytoid cells (Courtesy: Medicine Key).

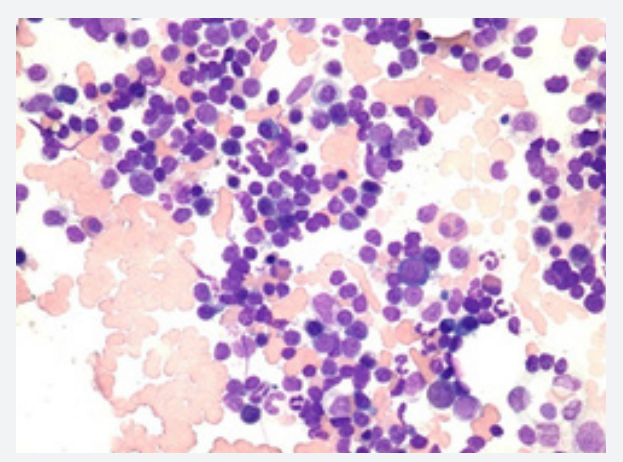

Figure 7: LPL inter-trabecular infiltrate in the bone marrow of plasmacytoid cells (Courtesy: Atlas of genetics and cytogenetics in oncology and haematology).

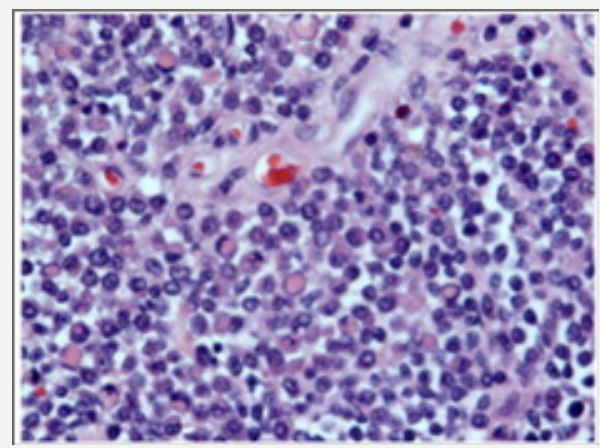

Figure 8: LPL lymphoid cells and plasmacytoid cells invading the central nervous system (Courtesy: Science direct).

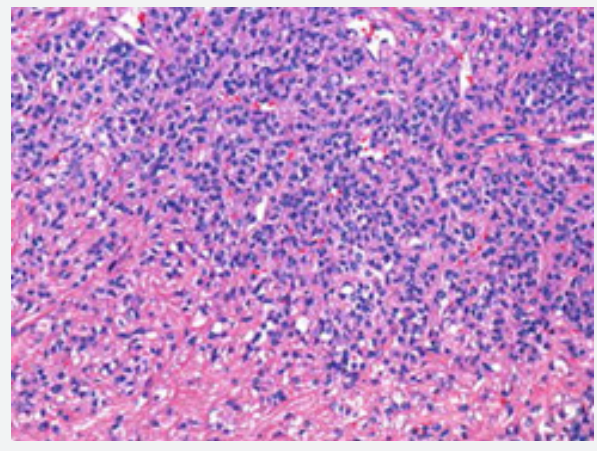

Figure 9: LPL tissue dispersal of lymphoid and plasmacytoid cells (Courtesy: Springer link).

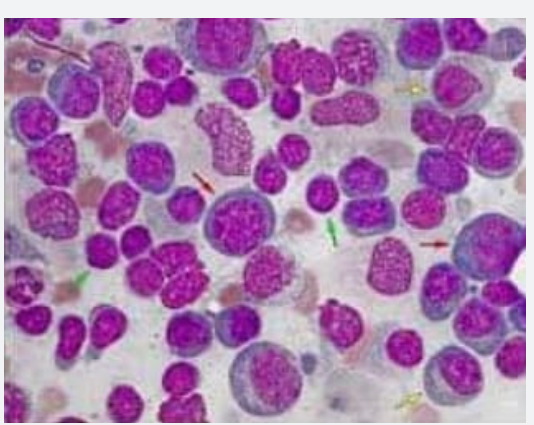

Figure 10: LPL plasmacytoid cells with open chromatin along with lymphoid cells with plasma cells (Courtesy: Leukaemia cell. org).

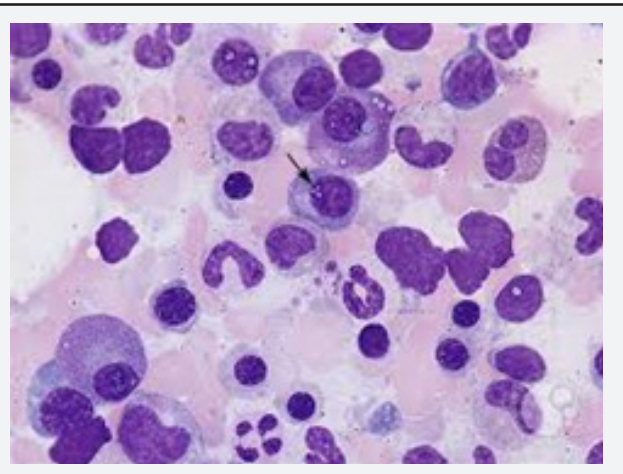

Figure 11: WM the presence of intra-nuclear inclusions termed as Dutcher's body (Courtesy: Image bank haematology).

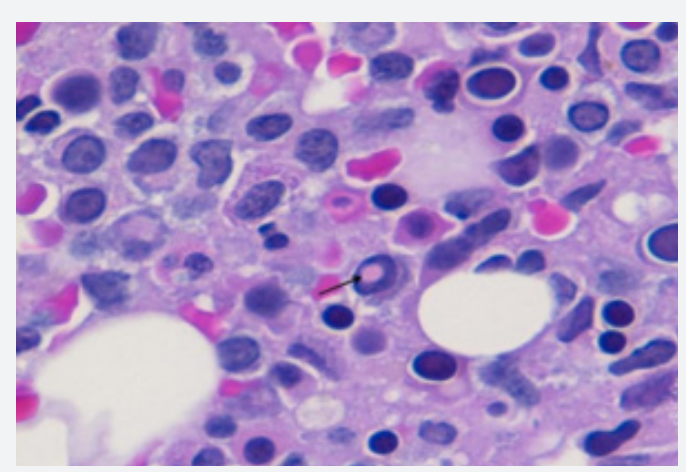

Figure 12: WM intra-nuclear inclusions known as Dutcher's body (Courtesy: Image bank haematology). 


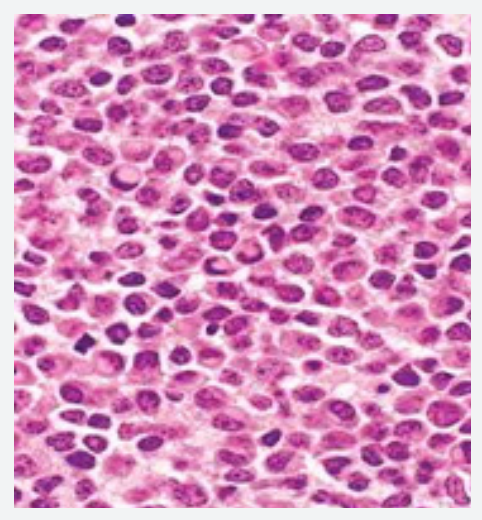

Figure 13: WM tissue dispersal of plasmacytoid cells with intranuclear Dutcher's body (Courtesy: Cambridge University Press).

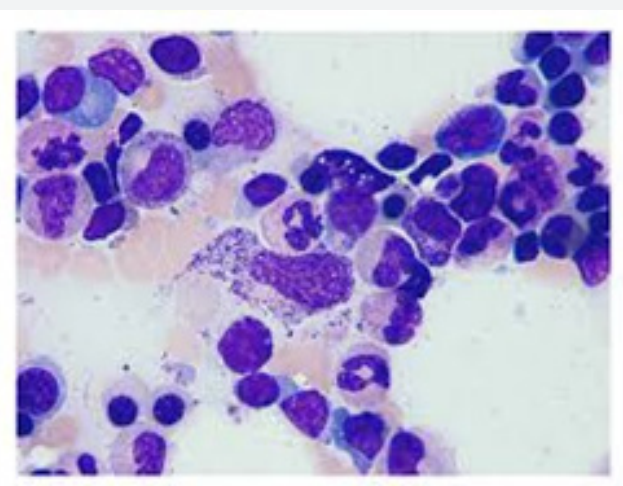

Figure 14: LPL lymphoid and plasmacytoid cells abutting the marrow trabeculae (Courtesy: Research Gate).

Criterion for Discerning Variants of Waldenstrom Macroglobulinaemia

1. A monoclonal gammopathy with Immune Globulin M (IgM) irrespective of the magnitude of M protein and an infiltration of lymphoplasmacytic cells greater than $10 \%$ in the bone marrow may be elucidated. Particularly inter-trabecular tumour dissemination may comprise of miniature lymphocytes with a plasmacytoid or plasma cell differentiation and a characteristic immune phenotype of immune reactive surface immune globulin $\mathrm{M}(\mathrm{Ig} \mathrm{M}+)$, CD19+, CD20+ and nonreactivity for CD5-, CD10-and CD23. The aforementioned evaluation may competently exclude adjunctive lympho proliferative disorders such as Chronic Lymphocytic Leukaemia (CLL) and mantle cell lymphoma $[1,2]$.

2. A monoclonal gammopathy of undetermined significance (Ig M MGUS) may enunciate serum monoclonal immunoglobulin M (Ig M) protein values below 3 grams/ decilitre with a lymphoplasmacytic cellular infiltrate beneath $10 \%$ generally confined to the bone marrow. Systemic symptoms of anaemia, hyper-viscosity, lymph node enlargement or hepato-splenomegaly may be absent. Immunoglobulin $\mathrm{M}$ monoclonal gammopathy of undetermined significance (Ig M MGUS) may evolve into a florid Waldenstrom macroglobulinaemia or an adjunctive B lymphocyte malignancy. The proportion of malignant transformation may emerge at an estimated $1.5 \%$ every year.

3. Smouldering Waldenstrom macroglobulinaemia may be an indolent or asymptomatic disorder. Monoclonal serum protein values for immunoglobulin M (Ig M) exceeding 3 grams/decilitre and/or a lymphoplasmacytic infiltrate restricted to the bone marrow in excess of $10 \%$ may be enunciated. End organ damage with coexistent anaemia, hyper-viscosity, lymph node enlargement or hepato-splenomegaly on account of a lymphoplasmacytic proliferation may be absent $[1,2]$.

\section{Investigative Assay}

Discernment of Waldenstrom macroglobulinaemia mandates a disease evaluation with complete blood count, serum chemistries such as liver and renal function tests, blood glucose and specific procedures such as bone marrow trephine biopsy and bone marrow aspiration. Serum immunoglobulin assay may depict an overproduction of immunoglobulin $\mathrm{M}$ with a decline in the values of immunoglobulin G and A (Ig G and I $\mathrm{gA}$ ), feature which may enhance the probability of emergent infections. Radiographic investigations may include a plain X-ray, Computerized Tomography (CT) scan, a Magnetic Resonance Imaging (MRI), an ultrasound and Positron Emission Tomography (PET) scan of the lymph node enlargement, enlarged spleen and dermal or tissue infiltrates of lymphoplasmacytic lymphoma cells $[9,10]$. Ocular examination may incorporate the assessment of retina and ocular fundus.

\section{Therapeutic Options}

Commencement of therapeutic intervention may concur with the appearance of B symptoms such as fever, night sweats, weight loss, fatigue, lymph node enlargement or splenomegaly. Haemoglobin declining to below 10 grams/decilitre or a platelet count below 100,000/ microlitre may be cogitated on account of bone marrow infiltration. Additionally, complications such as hyper viscosity syndrome, symptomatic sensory or motor peripheral neuropathy, systemic amyloidosis, renal insufficiency or symptomatic cryoglobulinaemia may require therapy $[10,11]$. A policy of careful observation may suffice for the indolent disorder. An estimated half (50\%) of the subjects with Waldenstrom macroglobulinaemia devoid of constitutional symptoms and a lack of treatment at 3 years following diagnosis may be managed with "active surveillance ". Approximately $10 \%$ instances may not require a therapeutic intervention during a 10year duration following detection. It may be crucial to establish the emergence of a hyper-viscosity syndrome prior to initiation of therapy and if plasmapheresis may be necessitated. Waldenstrom macroglobulinemia when associated with hyper-viscosity may display systemic symptoms such as visual deterioration, neurological symptoms and haemorrhage, 
incurring with immunoglobulin $M$ serum values greater than 4 grams/decilitre and the condition may be benefitted with plasmapheresis [11,12]. Chemotherapeutic agents found efficacious in Waldenstrom macroglobulinaemia include chlorambucil, cyclophosphamide, bendamustine, nucleoside analogues such as fludarabine and cladribine. Corticosteroids prednisone or dexamethasone may be applicable. Biologic therapy may enunciate the utilization of anti monoclonal antibody conjugates such as rituximab, ofatumumab or obinutuzumab. Immune modulators such as thalidomide and lenalidomide may prove to be effective [1,2]. Administration of proteasome inhibitors such as bortezomib, carfilzomib and ixazomib may be advantageous. Targeted therapy implicating the B cell signalling pathway may concur as imbruvica and everolimus [12,13]. Initial or preliminary therapy for previously untreated, symptomatic subjects may employ

a. purine analogues with a combination of fludarabine, cyclophosphamide and rituximab (FCR) or fludarabine and rituximab (FR).

b. Alkylating agents in varying combinations such as rituximab with cyclophosphamide, doxorubicin, vincristine and prednisone (R CHOP), dexamethasone, rituximab and cyclophosphamide (DRC) or rituximab with bendamustine (BR) may be beneficial.

c. Bortezomib in diverse combinations such as bortezomib, dexamethasone and rituximab (BDR) may be applicable.

d. Singular antibody conjugate such as rituximab may be utilized for initiation of therapy.

e. Ibrutinib as a Bruton's tyrosine kinase inhibitor (BTK inhibitor) may be efficacious in instances with MYD88 mutation. Concomitant chemo immunotherapy may be administered.

Administration of bendamustine with rituximab may depict a median and inter-quartile range (IQR) of 69.5 months in patients of Waldenstrom macroglobulinaemia [1,2]. The application of R CHOP may display a median and inter-quartile range (IQR) of 28.1 months. Solitary administration of rituximab in Waldenstrom macroglobulinemia may exhibit an objective response rate (ORR) of $52 \%$, a partial response (PR) of $27 \%$ and a minor response (MR) of $25 \%$. The median duration of response (DOR) may be elucidated at 27 months. An estimated half (54\%) of the individuals may depict an elevated immunoglobulin $\mathrm{M}$ (Ig M) "flare" and one fourth (27\%) subjects may delineate a persistent elevation of serum immunoglobulin $M$ at 4 months duration following discernment of disease. Administration of ibrutinib may demonstrate an objective response rate (ORR) of $90.5 \%$, a partial response (PR) of $73 \%$ and the median time to suitable therapeutic response may appear at 4 weeks. The progression free survival (PFS) at the end of 2 years may be
69.1\% and Overall Survival (OS) at $95.2 \%$. Toxicity levels to the chemotherapeutic agent may be greater than grade 2 . Ibrutinib administration may be accompanied by specific toxicities such as thrombocytopenia, neutropenia, stomatitis, atrial fibrillation, diarrhoea, herpes zoster infection, haematoma, secondary hypertension and epistaxis [13].

\section{Contemporary Instances}

Contemporary instances of Waldenstrom macroglobulinaemia may necessitate management as described:

1. Monoclonal gammopathy of undetermined significance (Ig M MGUS) with a lymphoplasmacytic infiltrate below $10 \%$, an asymptomatic or smouldering Waldenstrom macroglobulinaemia with haemoglobin greater than 11 grams/decilitre, a platelet count in excess of 120,000/ millilitre may be managed by" watchful waiting".

2. A symptomatic Waldenstrom macroglobulinaemia with a haemoglobin level below 11 grams/decilitre or a platelet count beneath 120,000/ millilitre.

a. Immunoglobulin M (Ig M) related neuropathy.

b. Haemolytic anaemia secondary to Waldenstrom macroglobulinaemia.

c. Symptomatic cryoglobulinaemia. The aforementioned instances may be managed with a solitary antibody conjugate such as rituximab. A maintenance therapy may not be required. Plasmapheresis may be opted for in instances of hyper-viscosity secondary to chemotherapy $[1,2]$.

3. Waldenstrom macroglobulinaemia elucidating bulky disease (tumour magnitude greater than 10 centimetres) or profound pancytopenia with reduced blood counts such as haemoglobin below 10 grams/decilitre or a platelet count beneath $100,000 /$ millilitre with the appearance of constitutional symptoms and a lack of features of hyper-viscosity or hyper-viscosity may be managed with plasmapheresis.

The aforementioned subjects may be administered concomitant bendamustine with rituximab with the regimen devoid of maintenance therapy with singular rituximab. Stem cell transplantation may be a pre-requisite. Alternatively, stem cells may be mobilized and cryo-preserved for subjects beneath $\leq 60$ years of age or emerge as potential and future candidates of Autologous Stem Cell Transplantation (ASCT). Stem cell transplantation may be suitably employed with subjects of relapsed or refractory Waldenstrom macroglobulinaemia. Autologous stem cell transplant (ASCT) may depict a 5year Progression Free Survival (PFS) of $40 \%$ and an overall survival (OS) of $68 \%$. Allogeneic stem cell transplant may exhibit a 5 year progression free survival (PFS) of $56 \%$ and a 5 year overall survival (OS) of $62 \%[1,2]$. Clinical trials with novel agents or 
drug conjugates may be mandated. Contemporary drugs such as ibrutinib, a Bruton's tyrosine kinase inhibitor or idelalisib, a PI3kinase inhibitor or everolimus, an m TOR inhibitor may be efficaciously adminstered. Contemporary anti CD20 antibody conjugates such as of tamumab, anti BCL2 agents such as venetoclax, recent histone deacetylase (HDAC) inhibitors such as panobinostat, recent proteasome inhibitor carfilzomib, recent immunomodulatory agents such as pomalidomide may be additionally assessed. Contemporary targeted therapies may include molecules such as ventoclax, acalabrutinib and BGB3111. The aforementioned drugs may be utilized in combination with established agents.

\section{Salvage Therapy}

Salvage Therapy may be applicable in specific instances. In subjects where the requirement of subsequent therapy may exceed 4 years, the original therapeutic regimen may be replicated. For therapeutic installation within 4 years, a monotherapy with ibrutinib or combinations such as Dexamathasone, Rituximab and Cyclophosphamide (DRC) may be opted for. Concomitant administration of bendamustine with rituximab (BR), Bortezomib, Dexamethasone and Rituximab (BDR) may be effective.

\section{Supportive Therapy}

Supportive Therapy may incorporate modalities such as blood transfusion, administration of growth factors in order to enhance the blood cell counts (white and red blood cells with platelets). Surgical procedures may be specified in particular instances in the form of splenectomy or plasmapheresis in order to reduce the serum immunoglobulin M (Ig M) quantities. Targeted radiation may be employed in order to decimate the magnitude of incriminated lymph nodes $[12,13]$ Table 1.

Table 1: Distinction betwixt Lymphoplasmacytic Lymphoma (LPL) and small cell Plasma Cell Myeloma (PCM) $[1,2]$.

\begin{tabular}{|c|c|}
\hline LPL & Small cell PCM \\
\hline Ig M para-protein may emerge & Para-protein may be non Ig M \\
\hline Surface Immunoglobulin may be monoclonal & Surface immunoglobulin may be minimal or absent \\
\hline Immune reactive CD138+ subset & Comprehensive immune reactivity to CD138+ \\
\hline Immune reactive CD19+, CD45+, PAX5+ subset & Tumour cells may be nonreactive to CD45-, CD19-, PAX5- \\
\hline Cyclin D1 nonreactive & Immune reactive to cyclin D1+ \\
\hline Mutation of MYD88 & Mutation of MYD88 wild type \\
\hline
\end{tabular}

\section{References}

1. Stephen M Ansell (2018) Update on Waldenstrom's macroglobulinemia. Leukaemia and Lymphoma Society.

2. International Waldenstrom's macroglobulinemia Foundation. About Waldenstrom's macroglobulinemia Factsheet 2018.

3. Rosai, Ackerman's (1986) Surgical Pathology.

4. Buske C, Leblond V (2013) Waldenstrom's macroglobulinemia- ESMO clinical practice guidelines for diagnosis, treatment and follow up. Ann Oncol 24 Suppl 6: vi155-vi159.

5. Gertz MA (2017) Waldenstrom macroglobulinemia: 2017 update on diagnosis, risk stratification and management. Am J Haematol 92(2): 209-217.

6. National Comprehensive Cancer Network (NCCN) Clinical practice guidelines in oncology "Waldenstrom's macroglobulinemia/ Lymphoplasmacytic Lymphoma “ V.1.2018.

7. Castillo JJ, D Sa S, Lunn MP, Minnema MC, Tedeschi A, et al. (2016) Central nervous system involvement by Waldenstrom's macroglobulinemia (Bing -Neel Syndrome): a multi centric retrospective study. Br J Haematol 172(5): 709-715.
8. Cao X1, Medeiros LJ, Xia Y, Wang X, Thomas SK, et al. (2016) Clinicopathologic features and outcomes of lympho-plasmacytic lymphoma patients with Ig G and Ig A paraprotein expression. Leuk Lymphoma 57(5): 1104-1113.

9. Shi M, Spurgeon S (2015) MYD88 analysis of a rare composite chronic lymphocytic leukaemia and lympho-plasmacytic lymphoma by flow cytometry cell sorting. Ann Haematol 94(11): 1941-1944.

10. Bassarova A, Trøen G, Spetalen S, Micci F, Tierens A, et al (2015) Lympho-plasmacytic lymphoma and marginal zone lymphoma in the bone marrow: paratrabecular involvement as an important distinguishing feature. Am J Clin Pathol 143(6): 797-806.

11. Oza A, Rajkumar SV (20115) Waldenstrom macroglobulinemia: prognosis and management. Blood Cancer J 27(5): e394.

12. Gertz MA (2018) Waldenstrom macroglobulinemia: treatment algorithm 2018. Blood Cancer J 8(4): 40.

13. Naderi N, Yang DT (2013) Lympho-plasmacytic lymphoma and Waldenstrom macroglobulinemia. Arch Pathol Lab Med 137(4): 580585 . 


\section{Your next submission with Juniper Publishers will reach you the below assets}

- Quality Editorial service

- Swift Peer Review

- Reprints availability

- E-prints Service

- Manuscript Podcast for convenient understanding

- Global attainment for your research

- Manuscript accessibility in different formats ( Pdf, E-pub, Full Text, Audio)

- Unceasing customer service

Track the below URL for one-step submission https://juniperpublishers.com/online-submission.php 